\title{
Cardiovascular impairment in a patient with acute myelitis
}

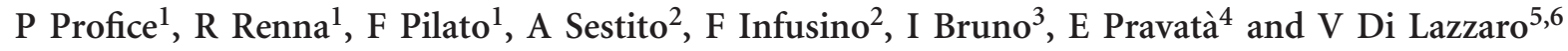

Study design: Case report.

Objective: to report and discuss the development of sudden symptomatic sinus bradycardia in a 35-year-old woman with acute myelitis.

Case report: A 35-year-old woman presented rapidly progressive weakness and hypoesthesia in the left hemibody. Five days after symptom onset, she developed symptomatic sinus bradycardia up to 30 b.p.m. Bradycardia was completely resolved $\sim 36 \mathrm{~h}$ after its onset.

Results: Cervical spine magnetic resonance imaging showed a focal T2-hyperintense intramedullary lesion at C2 level, with moderate cord swelling. The lesion involved bilaterally dorsal funiculi, and left lateral and ventral funiculi. Cardiac I-123 metaiodobenzylguanidine (MIBG) scintigraphy showed a decreased cardiac MIBG uptake suggesting sympathetic denervation.

Conclusion: The most likely explanation for bradycardia in our patient is the myelitis-related disruption of descending vasomotor pathways, resulting in sympathetic hypoactivity. Our case extends the spectrum of the clinical presentations of cervical myelitis and emphasizes the importance of careful cardiac monitoring in acute phase of cervical myelitis.

Spinal Cord (2013) 51, 511-513; doi:10.1038/sc.2013.30; published online 16 April 2013

Keywords: myelitis; symptomatic sinus bradycardia; cardiac scintigraphy

\section{INTRODUCTION}

It is well known that traumatic spinal cord injury is frequently complicated by cardiocirculatory impairment, ${ }^{1}$ while it is unknown whether the same phenomenon can manifest in acute myelitis. Here we describe a young woman presenting with acute cervical myelitis complicated by symptomatic bradycardia episodes.

\section{CASE REPORT}

A 35-year-old woman presented occipital headache followed by progressive left upper limb weakness, and hypoesthesia of the left hemibody. Her vital signs at initial evaluation at the emergency department were: 70 b.p.m. pulse rate, $100 / 70 \mathrm{~mm} \mathrm{Hg}$ blood pressure, and a respiratory rate of 15 breaths $\min ^{-1}$ with $99 \%$ oxygen saturation while breathing room air and normal temperature $\left(36.5^{\circ} \mathrm{C}\right)$; the neurologic examination revealed left lateropulsion at Romberg test, mild weakness in the left arm and hypoesthesia in the left hemibody with a C2 upper level. Cervical spine magnetic resonance imaging (MRI) showed a focal T2-hyperintense intramedullary lesion at C2 level, with moderate cord swelling. The lesion extended across more than two-thirds of the cord thickness, involving both the dorsal funiculi, and the left lateral and ventral funiculi. The dorsal aspect of the lateral funiculi represents the localization of the descending vasomotor pathways (DVPs) (Figure 1). Brain MRI demonstrated no pathological findings. Laboratory investigations revealed normal complete blood cell count as well as normal basic metabolic panel. Extensive laboratory investigations (inflammatory markers, thrombophilic and autoimmunity tests) were normal. Neurotropic virus serology in serum and cerebrospinal fluid (CSF) was negative for acute infections. CSF analysis revealed no changes in glucose and protein levels, no cells and negative oligoclonal bands. The patient was diagnosed with acute myelitis of uncertain etiology and started on a course of high-dose intravenous (i.v.) methylprednisolone $(1000 \mathrm{mg}$ per die for 8 days), with an initial improvement in her clinical status. I.v. corticosteroid therapy was followed by oral corticosteroids $(50 \mathrm{mg}$ prednisone per die with gradual tapering). During the course of hospitalization, 5 days after the onset of symptoms, the patient developed symptomatic sinus bradycardia up to 30 b.p.m. Cardiac enzymes were within normal values. Electrocardiograms recorded during the symptomatic bradycardia episodes revealed sinus bradycardia (minimum heart rate 35b.p.m.) and prolonged QT interval (QT/QTc: $570 / 486 \mathrm{~ms}$ ). The patient was successfully treated with a single i.v. administration of $0.5 \mathrm{mg}$ of atropine followed by i.v. dopamine $\left(2 \mathrm{mcg} \mathrm{kg}^{-1} \mathrm{~min}^{-1}\right)$ infusion. Prophylactic patch electrodes were positioned on the patient's chest for eventual non-invasive transcutaneous cardiac pacing. About $6 \mathrm{~h}$ later dopamine infusion was stopped and chest patch electrodes were removed, as the heart rate was $45-50$ b.p.m. and the patient was asymptomatic. Bradycardia was completely resolved about $36 \mathrm{~h}$ after its onset. In the acute period, the patient did not experience significant changes in blood pressure values. Serial transthoracic echocardiograms revealed no pathological findings. Heart MRI showed normal ventricular wall thickness with normal global and regional ventricular systolic function. A cardiac I-123 metaiodobenzylguanidine (MIBG) scintigraphy was performed. Anterior planar scintigraphy images were obtained $15 \mathrm{~min}$ (early) and $3 \mathrm{~h}$ (delayed) after $185 \mathrm{MBq}$ i.v. 123I-MIBG injection. MIBG uptake was semiquantified by calculating a heart-to-mediastinum (H/M)

${ }^{1}$ Institute of Neurology, Rome, Italy; ${ }^{2}$ Institute of Cardiology, Rome, Italy; ${ }^{3}$ Institute of Nuclear Medicine, Catholic University Rome, Rome, Italy; ${ }^{4}$ Institute of Radiology, Catholic University Rome, Rome, Italy; ${ }^{5}$ Institute of Neurology, Campus Bio-Medico University, Rome, Italy and ${ }^{6}$ Fondazione Alberto Sordi - Research Institute for Ageing, Rome, Italy Correspondence: Professor V Di Lazzaro, Institute of Neurology Campus Bio-Medico University, UniversitÃ campus Biomedico, Via Alvaro del Portillo, 200, Rome 00128, Italy. E-mail: v.dilazzaro@unicampus.it

Received 22 November 2012; revised 1 March 2013; accepted 16 March 2013; published online 16 April 2013 
a

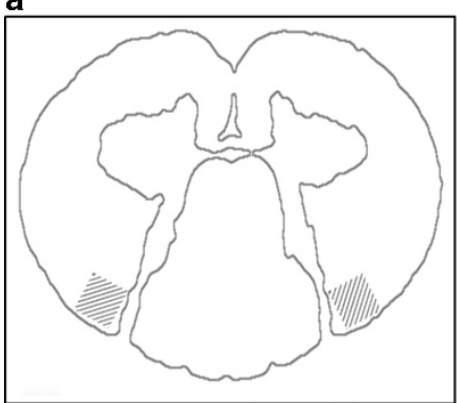

b

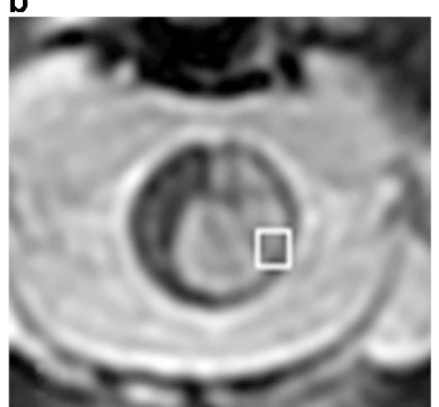

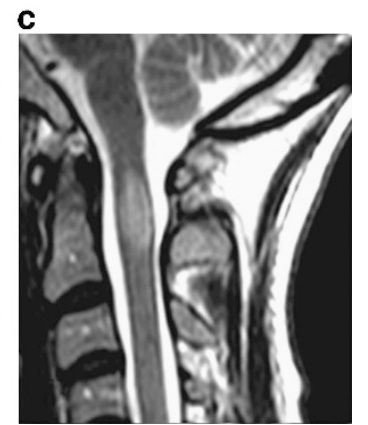

Figure 1 (a) Schematic diagram of the spinal cord (radiological orientation): dashed areas in the dorsal aspect of the lateral funiculi indicate the DVP localizations in the dorsal aspects of the lateral funiculi. (b, c) Patient's axial and sagittal T2-weighted fast-spin-echo MRI images demonstrate moderate cervical cord swelling and signal hyperintensity at C2 level involving the left DVP location in the dorsal aspect of the left lateral funiculus (boxed area in b).

a

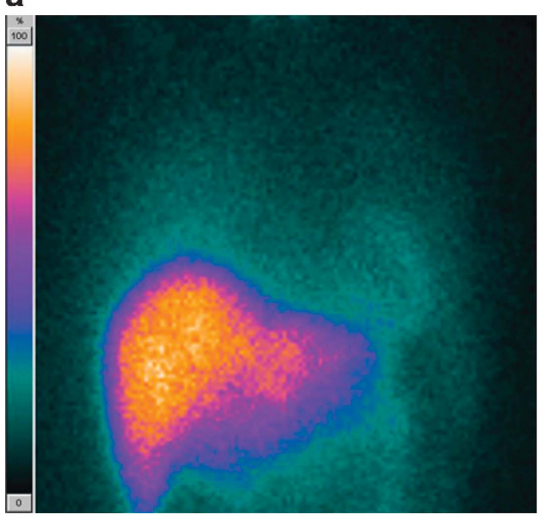

c

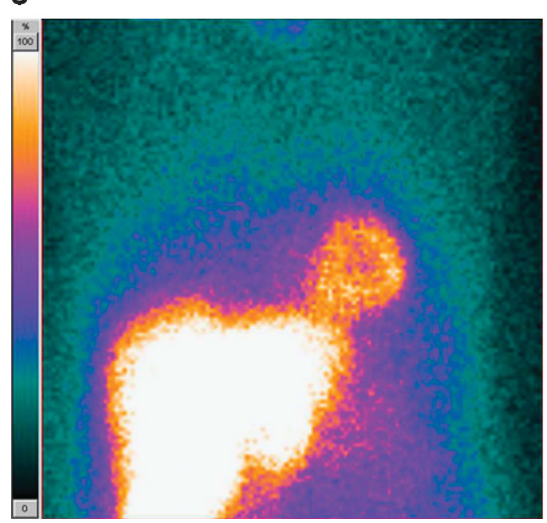

b

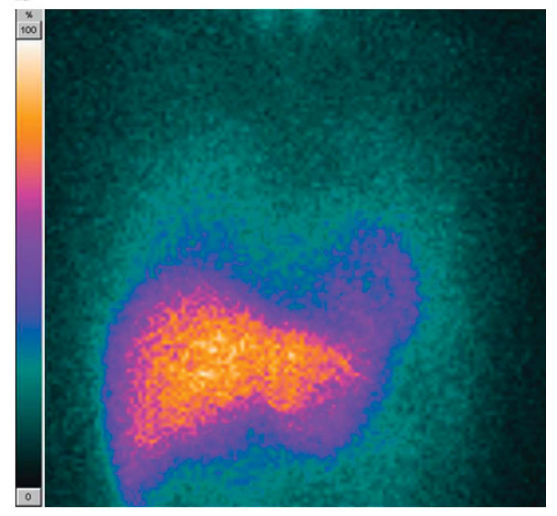

d

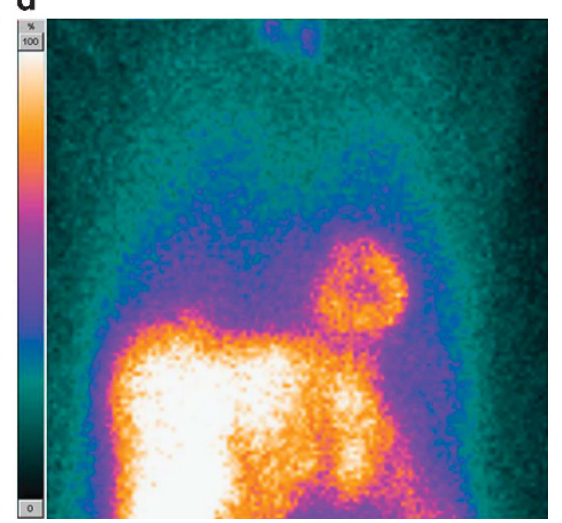

Figure 2 Cardiac MIBG scintigraphy. A low early (a) and delayed (b) MIBG H/M ratio is observed in the patient. Planar anterior images of a normal cardiac MIBG scintigraphy of an age and sex-matched control, with no cardiac pathology (c, d).

ratio, after drawing regions-of-interest (ROIs) over the heart and the upper mediastinum in the planar anterior view. Average counts per pixel in the myocardium were divided by average counts per pixel in the mediastinum. MIBG scintigraphy showed abnormal early and delayed H/M ratio: 1.6 and 1.3, respectively (normal value in our laboratory $2.2 \pm 0.3)^{2,3}$ (Figure 2).

\section{DISCUSSION}

Three spinal cord structures involved in cardiovascular control have been identified: DVPs, sympathetic preganglionic neurons and spinal afferents. The DVP is supposed to be in the dorsal aspect of the lateral funiculi ${ }^{4}$ and was involved on the left side by the spinal cord inflammatory lesion in our patient (Figure 1). The most likely explanation for bradycardia in our patient is the myelitis-related disruption of descendent pathways from the brainstem cardiovascular center to spinal sympathetic neurons into intermediolateral nuclei of T1-L2 segments, resulting in sympathetic hypoactivity. Our hypothesis is also confirmed by myocardial MIBG scintigraphy. This technique permits an objective evaluation of cardiac sympathetic function. ${ }^{5}$ MIBG is a norepinephrine analog with no pharmacological activity. It competes with norepinephrine for the same cellular transporter mechanisms of postganglionic adrenergic neurons. Like 
norepinephrine, MIBG is actively transported into noradrenaline granules of sympathetic nerve terminals by the noradrenaline transporter. Because of its affinity to sympathetic nerve endings MIBG accumulates in organs with a high sympathetic activity, such as adrenal gland, heart, liver and spleen. For diagnostic purposes MIBG is marked by a radioactive isotope, such as 123 -iodine. MIBG accumulation is visualized and measured by planar whole-body scintigraphy, permitting the visualization of catecholaminergic innervations in vivo. Although our patient had a central lesion and not a postganglionic lesion, MIBG scintigraphy revealed a decreased cardiac uptake of MIBG (Figures $2 \mathrm{a}$ and b), suggesting cardiac sympathetic denervation. Sympathetic hypoactivity has been reported in acute spinal lesions in animal studies ${ }^{6}$ with reversible changes of sympathetic preganglionic neurons during the first week after cord transection. $^{7-9}$ Acute changes of the sympathetic preganglionic neurons with later morphological recovery have also been reported in human studies. ${ }^{10}$ However, in animal studies the observed changes disappeared in 2-4 weeks, while in our case there was a full clinical recovery, suggestive of a sympathetic activity normalization, in only $36 \mathrm{~h}$. In our patient the lesion was incomplete and of inflammatory nature; thus, it can be hypothesized that there was a large proportion of axons with a short-lasting conduction block that promptly recovered. In any case, the reason for such a short-lasting change we observed remains largely unexplained. It should also be considered that the changes in preganglionic neurons reported in experimental studies $^{7-9}$ cannot fully explain the changes in postganglionic activity suggested by MIBG in our patient. One possibility is that the spinal cord lesion had an effect on neurotransmission of postganglionic sympathetic neurons innervating the heart producing a failure in norepinephrine release. A similar phenomenon has been reported in experimental studies showing a failure of neuromuscular junction transmission for skeletal muscles a few days after spinal cord injury. ${ }^{11}$

\section{CONCLUSIONS}

Our case extends the spectrum of the clinical presentations of cervical myelitis and emphasizes the importance of careful cardiac rhythm monitoring in the acute phase of cervical myelitis. This is the first MIBG study suggesting short-lasting sympathetic denervation of the heart in a patient with an acute high cervical cord lesion; further studies are needed to evaluate the possible role of MIBG studies in patients with spinal cord lesion and to better understand the observed functional changes.

\section{CONFLICT OF INTEREST}

The authors declare no conflict of interest.

1 Popa C, Popa F, Grigorean VT, Onose G, Sandu AM, Popescu M et al. Vascular dysfunctions following spinal cord injury. J Med Life 2010; 3: 275-285.

2 Di Monaco A, Lanza GA, Bruno I, Careri G, Pinnacchio G, Tarzia P et al. Usefulness of impairment of cardiac adrenergic nerve function to predict outcome in patients with cardiac syndrome X. Am J Cardiol 2010; 106: 1813-1818.

3 Flotats A, Carrió I, Agostini D, Le Guludec D, Marcassa C, Schäfers M et al. EANM Cardiovascular Committee; European Council of Nuclear Cardiology. Proposal for standardization of 123I-metaiodobenzylguanidine (MIBG) cardiac sympathetic imaging by the EANM Cardiovascular Committee and the European Council of Nuclear Cardiology. Eur J Nucl Med Mol Imaging 2010; 37: 1802-1812.

4 Furlan JC, Fehlings MG, Shannon P, Norenberg MD, Krassioukov AV. Descending vasomotor pathways in humans: correlation between axonal preservation and cardiovascular dysfunction after spinal cord injury. J Neurotrauma 2003; 20: 1351-1363.

5 Yamashina S, Yamazaki J. Neuronal imaging using SPECT. Eur J Nucl Med Mol Imaging 2007; 34 (Suppl 1): S62-S73.

6 Weaver LC, Fleming JC, Mathias CJ, Krassioukov AV. Disordered cardiovascular control after spinal cord injury (Chapter 13). Verhaagen J and McDonald JW III (eds). Handbook of Clinical Neurology, Vol. 109: Spinal Cord Injury, 3rd series Elsevier B.V.: Amsterdam, The Netherlands, 2012.

7 Krassioukov AV, Weaver LC. Morphological changes in sympathetic preganglionic neurons after spinal cord injury in rats. Neuroscience 1996; 70: 211-225.

8 Krenz NR, Weaver LC. Changes in the morphology of sympathetic preganglionic neurons parallel the development of autonomic dysreflexia after spinal cord injury in rats. Neurosci Lett 1998; 243: 61-64.

9 Llewellyn-Smith IJ, Weaver LC. Changes in synaptic inputs to sympathetic preganglionic neurons after spinal cord injury. J Comp Neurol 2001; 435: 226-240.

10 Krassioukov AV, Bunge RP, Pucket WR, Bygrave MA. The changes in human spinal sympathetic preganglionic neurons after spinal cord injury. Spinal Cord 1999; 37: 6-13.

11 Ollivier-Lanvin K, Lemay MA, Tessler A, Burns AS. Neuromuscular transmission failure and muscle fatigue in ankle muscles of the adult rat after spinal cord injury. $J$ Appl Physiol 2009; 107: 1190-1194. 